\title{
Correlations Between the Gradient of Contrast Density, Evaluated by Cardio CT, and Functional Significance of Coronary Artery Stenosis
}

\author{
Orzan Marius $^{1 *}$, Jako Beata ${ }^{1}$, Blendea Ciprian ${ }^{1,2}$, Benedek Annabell1, Bajka Balazs ${ }^{1}$, Benedek Imre \\ University of Medicine and Pharmacy of Tîrgu Mureș, Clinic of Cardiology, Tîrgu Mureș, Romania \\ 2 Cardio Med Medical Center, Department of Advanced Multimodal Imaging, Tîrgu Muress, Romania
}

\begin{abstract}
Background: Assessment of the hemodynamic significance of a coronary artery stenosis is a challenging task, being extremely important for the establishment of indication for revascularization in atherosclerotic coronary artery stenosis. The aim of this study was to evaluate the role of a new marker reflecting the functional significance of a coronary artery stenosis, represented by the attenuation degree of contrast density along the stenosis by Coronary CT. Material and Method: We evaluated retrospectively 30 patients with angina and coronary luminal narrowing, who underwent 64-slice Coronary Computed Tomography Angiography. We measured the stenosis degree, intraluminal contrast density (Hounsfield units [HU]) at two levels, proximal and distal to stenosis, and the attenuation gradient was calculated on this basis. Results: The average contrast density was $77,96 \mathrm{UH}$ proximal to the stenosis and 67,6 UH distal to the stenosis. The average transluminal gradient was 10,36. The average length of the coronary lesions was $16,93 \mathrm{~mm}$. In those lesions with significant stenosis, expressed by $>70 \%$ luminal narrowing, we recorded a significantly higher transluminal attenuation gradient as compared to those with $<70 \%$ luminal narrowing $(6.16+/-3.7$, $95 \% \mathrm{Cl} 4.3-80$ vs $16.6+/-8.4,95 \% \mathrm{Cl} 11.3-21.9)$. The degree of luminal narrowing significantly correlated with the contrast attenuation gradient $(r=0.71, p<0.001)$. Conclusions: The assessment of intraluminal contrast density by Coronary Computed Tomography Angiography may represent a new noninvasive tool to obtain relevant information about the clinical significance of a coronary stenosis. Larger studies are requested to emphasize the benefits brought by CCTA in evaluating coronary lesions.
\end{abstract}

Keywords: computer tomography, contrast density gradient,atherosclerosis, coronary lesions

Received: 18 August 2015 / Accepted: 02 November 2015

\section{Introduction}

Coronary artery disease remains a leading cause of death worldwide and still has an increasing prevalence. Therapeutic decision of revascularization depends not only on stenosis degree but also onlesion characteristics including its hemodynamic significance [1].

Development of imaging techniques including Coronary Computed Tomography Angiography offered the possibility of evaluating coronary arteries noninvasively $[2,3]$. With an improved spatial and temporal resolution, the 64-slice CT has an increasing role in indication for revascularization [4].

Currently there are several types of atherosclerotic lesions in whichthe strategy is not clear and the choice of treatment is difficult due to lack of clear recommendations, based on clear evidence, while the evolution of these patients largely depends on the type of treatment selected[5]. Such situations are recorded in case of coronary stenosis between $50 \%$ and $60 \%$ and also in case of the so-called non-significant $30-40 \%$ stenoses which associate typical symptoms or ECG changes.

The aim of our study was to evaluate a new imaging marker, represented by the degree of contrast attenuation along a coronary stenosis, thatcould provide functional

* Correspondence to: Marius Orzan

E-mail: orzan_marius@yahoo.com information aboutthe hemodynamic significance of coronary stenosis, based on assessment of the contrast density along the lesion using Angio CT.

\section{Material and Methods}

In this retrospective study we examined 30 consecutive patients who presented with chest pain in the period June 2014 - October 2014. Exclusion criteria from the study were renal insufficiency, allergy to contrast medium and unstable condition with incapacity to perform breath-hold. Based on reported symptoms and laboratory findings patients, were scheduled to undergo a Coronary Computed Tomography Angiography examination.

Two days before the examination any biguanide (oral antidiabetic medication) was stopped and oral Metoprolol LPH 25-50mg (LabormedPharma Romania) was given to achieve resting heart rate higher than 65 beats $/ \mathrm{min}$. An intravenous line was placed for contrast administration in the right antecubital vein.

The patients were divided into two groups, according to the severity of their coronary stenosis: group 1, with the degree of luminal narrowing $<70 \%$, and group 2 , with the degree of luminal narrowing $>70 \%$. Baseline characteristics of the study population showed no significant differences between the group with $>70 \%$ stenosis and the group with $<70 \%$ stenosis in terms of age ( $55.72 \mathrm{y}$ in gr 1 versus 59.66 y in group $2, p=0.22$ ), or risk factors (smok- 
ers, hypertension, dyslipidemia, $\mathrm{p}=\mathrm{ns}$ ). The only parameters that showed statistical significance at baseline was the presence of diabetes (Figure 1).

All examinations were performed using multi slice 64Somatom Sensation CT(Siemens, Germany). Used parameters were $64 \times 0.5 \mathrm{~mm}$ slice collimation, 0.33 gantry rotation time, tube current $390 \mathrm{mAs} /$ rotation, tube potential $120 \mathrm{mV}$. Coronary angiography scan was obtained with injection of $60 \mathrm{ml}$ of nonionic contrast medium (Iopamidol, Bayer HealthCare $370 \mathrm{mg} \mathrm{I} / \mathrm{ml}$ ) at a flow rate first with $4 \mathrm{ml} / \mathrm{s}$ followed by $20 \mathrm{ml}$ at $2.0 \mathrm{ml} / \mathrm{s}$. Scanning was made in the arterial phase using bolus tracking automatic contrast administration with the region of interest (ROI) set in the descending aorta. CT reconstructions were made to select the phase with least motion artifacts.

We measured the length of each stenoticlesion and the coronary intraluminal contrast density (Hounsfield units [HU]) at two levels: proximal and distal to the stenotic area. We calculated the attenuation gradient as the difference between the prestenoticand the poststenotic contrast density values (figure 2 ).

Our study was approved by the institutional review board of the clinical unit where the study was conducted and informed consent for using their data for the scope of the study was obtained from the patients.

\section{Results}

The average contrast density was $77.96 \mathrm{UH}$ at the level proximal to the stenosis and $67.6 \mathrm{UH}$ at the level distal to the stenosis. The average transluminal gradient was 10.36 and the average length of the coronary lesions was 16.93 $\mathrm{mm}$. There were no statistically significant differences between the groups in terms of the stenosis length $(17.83+/-$ $7.16 \mathrm{~mm}, 95 \%$ CI: $14.27-21.39$ versus $15.58+/-6.17 \mathrm{~mm}$, 95\% CI: 11.66-19.5, $\mathrm{p}=0.312$ ).

In those lesions with significant stenosis, expressed by $>70 \%$ luminal narrowing, we recorded a significantly higher transluminal attenuation gradient as compared to those with $<70 \%$ luminal narrowing $(6.16+/-3.7,95 \%$ CI: 4.3 80 vs. $16.6+/-8.4,95 \%$ CI: $11.3-21.9)$. The degree of luminal narrowing significantly correlated with the contrast attenuation gradient $(\mathrm{r}=0.71, \mathrm{p}<0.001)$ (Figure 3).

\section{Discussion}

Intraluminal contrast density gradient determination using Coronary Computed Tomography Angiography provides new information useful for complex characterization of the stenotic lesions located in the coronary arteries [6,7]. We observed a significantly higher contrast density gradient in case of significant stenosis with luminal narrowing $>70 \%$, as compared with the stenosis with luminal narrowing $<70 \%$. Our study demonstrates that in patients with significant stenosis, this gradient was $16.6+/-8.4$ compared to $6.16+/-3.7$ for the patients with less severe stenosis.

Like Nakanishi et al [8] we observe that the translesionalgradient brings new useful data regarding coronary artery

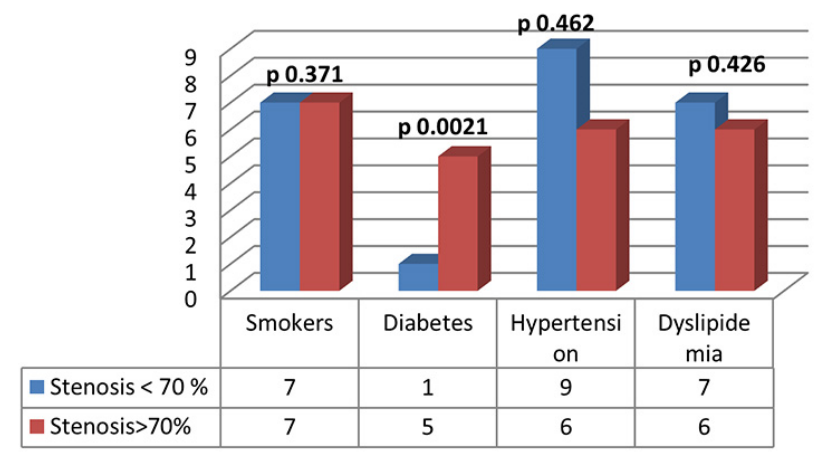

Fig. 1. The risk factors recorded in the two groups

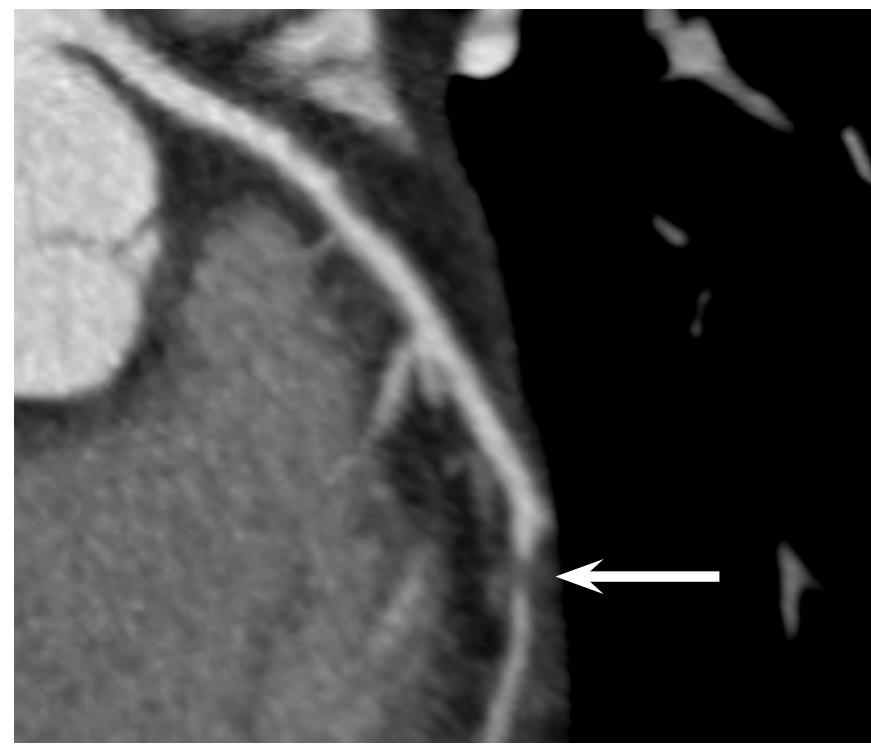

Fig. 2. Angio CT image, showing a $>70 \%$ stenosis caused by an atherosclerotic lesion in the middle part of the coronary artery

\section{Attenuation gradient}

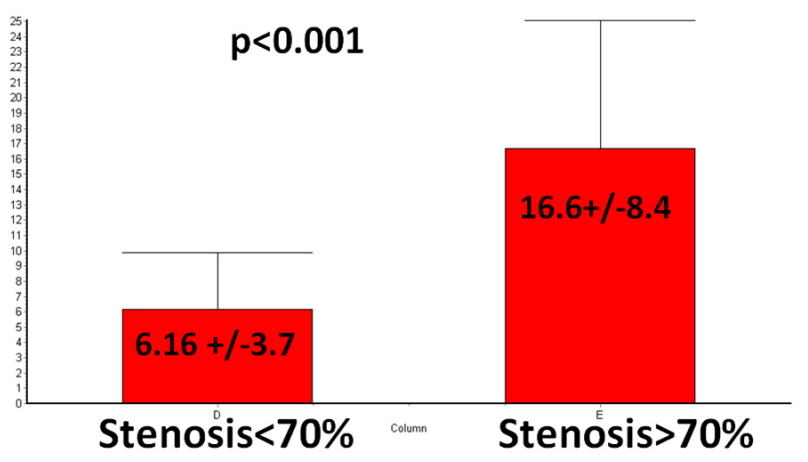

Fig. 3. The attenuation gradient along the coronary stenosis in the two groups of patients

stenosis. Also, this investigation does not require any additional explorations and it's not time consuming in comparison with the standard CCTO evaluation.

This difference is associated, in our opinion, with the hemodynamic impact of the stenotic lesion on the blood flow, that could trigger significant perturbances in the coronary that could change from laminar into turbulent. 
Flow changes could also accelerate the atherosclerotic process via increasing the action of hemodynamic forces on the atherosclerotic plaque. Based on a positive feed-back mechanism, it is possible that significant hemodynamic stenotic lesions could progress more rapidly than significant stenotic lesions without hemodynamic impact, which is reflected in our study by a lower intraluminal contrast density gradient.

Through a positive feed-back mechanism, it is possible for significant stenotic lesions to develop differently, in accordance to their hemodynamic significance, which is expressed in our study by the intraluminal contrast density gradient.

The sensitivity and specificity of this new marker, the intraluminal contrast density gradient, as it has been evaluated by Nakanishi $R$ et al. [8] seems to be high enough to be useful in differentiating coronary atherosclerotic lesions, according to their severity. Thus, FFRCT seems to be more accurate than classic CCTA in identifying coronary lesions and could be introduced in the future in the routine algorithm for characterization of the significance of coronary plaques.

Coronary Computed Tomography Angiography is a routine examination that represents an important step in the evaluation of patients with chest pain, according to the present guidelines. Intraluminal contrast density gradient determination for a coronary stenotic lesion is not a time consuming method, as it does not need another investigation beside the standard Coronary Computed Tomography Angiography. That is why it can be easily achieved in medical imaging centers with experience in Coronary Computed Tomography Angiography.

\section{Conclusion}

Intraluminal contrast density gradient is associated with the severity degree of the coronary stenosis and could represent a new marker useful for the complex assessment of the functional significance of a coronary lesion.

Further studies are required, on larger patient populations, for a more complex assessment of the role of this new marker in defining the functional significance of a coronary lesion. Also, follow-up studies are required in order to evaluate the correlation between MACE rates and the contrast density gradient along a coronary artery stenosis, as a measure of its significance.

\section{Acknowledgement}

This paper was published under the frame of European Social Found, Human Resources Development Operational Programme 2007-2013, project no. POSDRU/159/1.5/S/133377.

\section{Conflict of interest}

None to declare.

\section{References}

1. Hell MM, Dey D, Marwan M, et al. Non-invasive prediction of hemodynamically significant coronary artery stenoses by contrast density difference in coronary CT angiography.Eurupean Journal of Radiology.2015;84(8):1502-8.

2. Wong DT, Ko BS, Cameron JD, et al. Transluminal attenuation gradient in coronary computed tomography angiography is a novel noninvasive approach to the identification of functionally significant coronary artery stenosis: a comparison with fractional flow reserve. J Am CollCardiol. 2013;61(12):1271-9.

3. Choi JH, Koo BK, Yoon YE, et al.Diagnostic performance of intracoronary gradient-based methods by coronary computed tomography angiography for the evaluation of physiologically significant coronary artery stenoses: a validation study with fractional flow reserveEur Heart $J$ Cardiovasc Imaging. 2012;(12):1001-7.

4. BenedekImre,BenedekTheodora.Angio computertomografiemultiscliceîn diagnosticul bolilor cardiovasculare. Edidura Universității din Oradea. 2014

5. Al-Hassan D, Leipsic J. Noninvasive fractional flow reserve derived from coronary computed tomography angiography: integrated anatomical and functional assessment. Future Cardiol. 2013;9(2):243-51.

6. $\mathrm{Xu} \mathrm{L}$, Sun Z, Fan Z. Noninvasive physiologic assessment of coronary stenoses using cardiac CT. Biomed Res Int. 2015;2015:435737.

7. Stuijfzand WJ, Danad I, Raijmakers PG, et al. Additional value of transluminal attenuation gradient in CT angiography to predict hemodynamic significance of coronary artery stenosis. JACC CardiovasC Imaging. 2014;7(4):374-86

8. Nakanishi R, Matsumoto S, Alani A, et al. Diagnostic performance of transluminal attenuation gradient and fractional flow reserve by coronary computed tomographic angiography $(\mathrm{FFR}(\mathrm{CT}))$ compared to invasive FFR: a sub-group analysis from the DISCOVER-FLOW and DeFACTO studies. Int J Cardiovasc Imaging. 2015;31(6):1251-9. 